\title{
A CATEGORICAL GUIDE TO SEPARATION, COMPACTNESS AND PERFECTNESS
}

\author{
WALTER THOLEN \\ (communicated by Cristina Pedicchio)
}

\begin{abstract}
Based on a rather arbitrary class of morphisms in a category, which play the role of "closed maps", we present a general approach to separation and compactness, both at the object and the morphism levels. It covers essential parts of the classical topological theory, generalizes various previous categorical treatments of the theme, and allows for a number of less expected applications outside topology.
\end{abstract}

\section{Introduction}

The idea to define an object $X$ to be separated and compact by asking the natural "cooperations"

$$
X \longrightarrow X \times X \text { and } X \longrightarrow 1
$$

to belong to a given class $\mathcal{F}$ of morphisms in a category appears already in Penon's papers [Pe1], [Pe2], but does not seem to have been investigated much further at this level of generality, with the notable exception of the factorization-based approach of [HeSS] which in turn builds on Herrlich's early works [He1], [He2]. The essential question in this context is which conditions on $\mathcal{F}$ would allow for a satisfactory general theory of separation and compactness. While Penon assumes stability of $\mathcal{F}$ under pullback and under the formation of fibred products, we find that closure under composition is essential to fully exhibit the relationships between the two properties in question, but we take this to be our only condition on $\mathcal{F}$. However, we effectively enforce pullback stability by replacing $\mathcal{F}$ by its largest stable subclass $c(\mathcal{F})$; closure under fibred products is not used here since we mostly concentrate on finite properties in this paper.

It turns out that this approach not only yields a satisfactory theory of separation and compactness at both, the object and the morphism levels, but also of embeddable and of absolutelyclosed objects which, in topological terms, means: of Tychonoff spaces and of $H$-closed spaces. When we assume, in addition to our basic axiom that $\mathcal{F}$ be closed under composition, the existence of the analogue of the Stone-Čech compactification, we obtain at the abstract categorical $\mathcal{F}$-level analogues of the Isbell-Henriksen characterization of perfect maps between Tychonoff spaces (Theorem 4.8 ), and of the "constructive existence" of the antiperfect-perfect factorization, i.e., of the map version of the Stone-Čech compactification (Theorem 4.9).

Given the level of generality, it is not surprising that this approach covers a broad range of applications and special cases, only few of which we mention here in some detail. Within the realm of "topology", general studies of separation and compactness appeared previously in the context of closure operators, with a comprehensive recent account given in [CGT1]. Many of the techniques given in that paper re-appear here in greater generality, although the reader should notice the fact that separation as defined here may differ from Hausdorffness of [CGT1] for a closure operator which fails to be weakly hereditary (see 3.10). In any case,

Received 6 November 1998; published on 24 June 1999.

1991 Mathematics Subject Classification: 18B30, 18A40, 54B30

Key words and phrases: separated object, compact object, embeddable object, absolute object, perfect morphism

(C) 1999, Walter Tholen. Permission to copy for private use granted. 
the current paper covers not only the notions of separated and compact object w.r.t. a closure operator, but also that of a "discrete object" and of a "local homeomorphism" (see 2.5, 2.6). We also point out that, although topologically motivated, the closure-theoretic approach is by no means restricted to "topological" application; we mention here explicitly Diers' approach to algebraic sets $[\mathrm{Di}]$, in which separated and absolutely-closed objects occur most naturally (see 4.11).

We refer here to two other groups of examples which appeared recently in the literature. One is given by the decidable (or separable) objects and morphisms in extensive categories, as studied in [CJ]; they capture not only the topos-theoretic notion of decidability but also the notion of separable algebra (see 2.7). Extensitivity is also used in our general theory, in order to guarantee closure of separated and compact objects under finite sums (Theorem 3.8). The other group is given by separated objects and morphisms with respect to a pointed endofunctor, as presented recently in [JT]; this theory is closely connected with Janelidze's general approach to Galois- and covering theory [J]. Again, we exploit this "example" also for our general theory, since the factorization theorem 4.9 is derived from a result in [JT] which, in turn, generalizes the main result of [CHK].

Parts of the results contained in this paper were presented (under more restrictive conditions) in talks given by the author at the category theory meetings at Halifax (July, 1995), Antwerp (March, 1998) and Saint John (June, 1998), as summarized in the preliminary article [T2]. The author is grateful for various valuable comments received during these meetings, particularly from D. Bourn (for his direction to Penon's papers). He is also indebted to M. M. Clementino for providing the example given in 3.10.

\section{Stabilization and Derivation of a Class of Morphisms}

2.1 In a category $\mathcal{C}$ with finite limits, we fix a class $\mathcal{F}$ of morphisms which is always assumed to be closed under composition with isomorphisms. Denoting by $h^{*}(f)$ the pullback of $f$ along $h$, as in

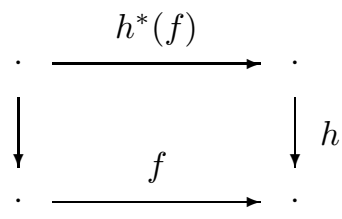

we form the class

$$
c(\mathcal{F}):=\left\{f \mid \forall h: h^{*}(f) \in \mathcal{F}\right\} .
$$

Then $c(\mathcal{F})$ is stable under pullback (in fact, it is the largest stable subclass of $\mathcal{F}$ ); in particular, $c(\mathcal{F})$ is left-cancellable w.r.t. monomorphisms (so that $m \cdot f \in c(\mathcal{F})$ with $m$ monic implies $f \in c(\mathcal{F}))$. Furthermore, $c(\mathcal{F})$ is closed under composition and contains all isomorphisms if $\mathcal{F}$ has the respective property.

2.2 For a morphism $f: X \rightarrow Y$, we denote by $\delta_{f}$ the diagonal of its kernelpair, that is : $\delta_{f}=<1_{X}, 1_{X}>: X \rightarrow X \times_{Y} X$, and we let

$$
d(\mathcal{F}):=\left\{f \mid \delta_{f} \in c(\mathcal{F})\right\} .
$$

Of course, when $\mathcal{F} \cap$ RegMonoC (with RegMonoC the class of regular monomorphisms of $\mathcal{C}$ ) is stable under pullback, the definition of $f \in d(\mathcal{F})$ simplifies to $\delta_{f} \in \mathcal{F}$, and $d(\mathcal{F})=\mathcal{F}^{\prime}$ as defined in [JT]. But the definition of $d(\mathcal{F})$ as given here enables us to derive all wanted properties without imposing conditions on $\mathcal{F}$. First, for $f: X \rightarrow Y$ and $g: Y \rightarrow Z$, one has pullback diagrams 


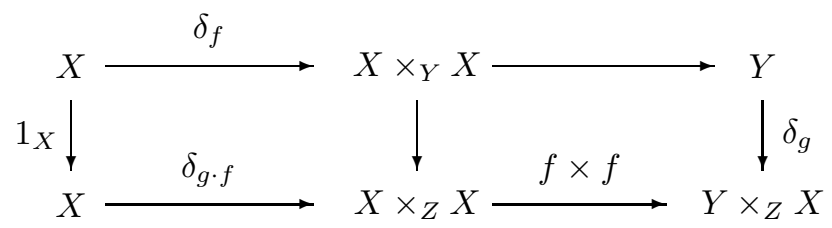

which give the formula $\delta_{g \cdot f} \cong(f \times f)^{*}\left(\delta_{g}\right) \cdot \delta_{f}$. Secondly, given the pullback on the right one has the pullback on the left in the following diagram:

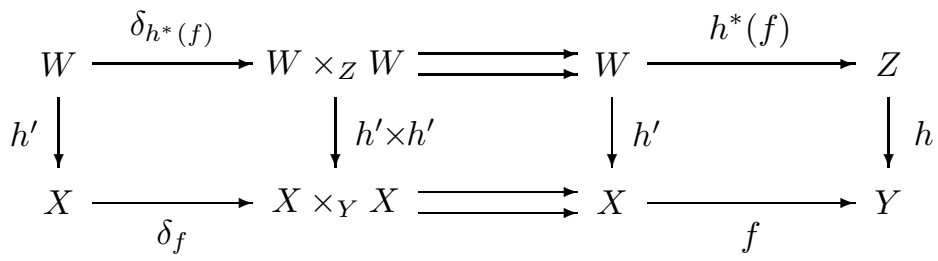

Hence, $\delta_{h^{*}(f)} \cong\left(h^{\prime} \times h^{\prime}\right)^{*}\left(\delta_{f}\right)$. With these formulas one sees immediately that $d(\mathcal{F})$ is pullbackstable and left-cancellable (with respect to all morphisms), and that $d(\mathcal{F})$ is closed under composition if $\mathcal{F}$ is; also, $d(\mathcal{F})$ contains all monomorphisms if $\mathcal{F}$ contains all isomorphisms.

2.3 The morphism class $\mathcal{F}$ naturally induces the class

$$
\mathcal{F}^{!}:=\left\{X \mid !_{X} \in \mathcal{F}\right\}
$$

of objects in $\mathcal{C}$; here $!_{X}: X \rightarrow 1$ is the unique morphism of the object $X$ into a (fixed) terminal object of $\mathcal{C}$. We can now form the classes

$$
\begin{array}{r}
C(\mathcal{F}):=c(\mathcal{F})^{!}=\left\{X \mid \forall Y:\left(p_{Y}: X \times Y \rightarrow Y\right) \in \mathcal{F}\right\}, \\
D(\mathcal{F}):=d(\mathcal{F})^{!}=\left\{X \mid\left(\delta_{X}: X \rightarrow X \times X\right) \in c(\mathcal{F})\right\} ;
\end{array}
$$

note that the projections $p_{Y}$ are precisely the pullbacks of $!_{X}$, and that we write $\delta_{X}$ instead of $\delta_{!_{X}}$. Properties of the classes $C(\mathcal{F})$ and $D(\mathcal{F})$ will be given in Section 3 .

Of course, while here we defined $C(\mathcal{F}), D(\mathcal{F})$ in terms of $c(\mathcal{F}), d(\mathcal{F})$, we could have proceeded conversely. The reason for this is the fact that "stabilization and derivation commute with slicing", as follows. For an object $B$ in $\mathcal{C}$, put

$$
\mathcal{F}^{B}=\left(\Sigma_{B}\right)^{-1}(\mathcal{F}),
$$

with $\Sigma_{B}: \mathcal{C} / B \rightarrow \mathcal{C}$ the forgetful functor of the category of $\mathcal{C}$-objects over $B$. Then

$$
c\left(\mathcal{F}^{B}\right)=c(\mathcal{F})^{B} \text { and } d\left(\mathcal{F}^{B}\right)=d(\mathcal{F})^{B},
$$

and in particular

$$
C\left(\mathcal{F}^{B}\right)=c\left(\mathcal{F}^{B}\right)^{!}=\left(c(\mathcal{F})^{B}\right)^{!} \text {and } D\left(\mathcal{F}^{B}\right)=d\left(\mathcal{F}^{B}\right)^{!}=\left(d(\mathcal{F})^{B}\right)^{!} .
$$

Hence, for $f: X \rightarrow Y$ in $\mathcal{C}$ one has

$$
f \in c(\mathcal{F}) \Longleftrightarrow(X, f) \in C\left(\mathcal{F}^{B}\right) \text { and } f \in d(\mathcal{F}) \Longleftrightarrow(X, f) \in D\left(\mathcal{F}^{B}\right) \text {. }
$$

2.4 For the sake of completeness we mention that both

$$
c, d: \operatorname{MORC} \rightarrow \operatorname{MORC}
$$

are right adjoint functors of the partially ordered "conglomerate" MORC of all morphism classes of $\mathcal{C}$ (which are closed under composition with isomorphisms). Their left adjoints assign 
to a class $\mathcal{G}$ the classes

$$
\overline{\mathcal{G}}=\left\{h^{*}(g) \mid h \in \operatorname{Mor} \mathcal{C}, g \in \mathcal{G}\right\} \text { and } \widetilde{\mathcal{G}}=\left\{h^{*}\left(\delta_{g}\right) \mid h \in \operatorname{Mor} \mathcal{C}, g \in \mathcal{G}\right\},
$$

respectively.

Also the passage $\mathcal{F} \longmapsto \mathcal{F}^{\text {! }}$ has a left adjoint: it assigns to every (isomorphism-closed) class $\mathcal{B}$ of objects in $\mathcal{C}$ the morphism class $\operatorname{Hom}(\mathcal{B}, 1)=\left\{!_{B} \mid B \in \mathcal{B}\right\}$. Composition with $c, d$ yields two right adjoints

$$
C, D: \mathrm{MORC} \rightarrow \mathrm{OBC}
$$

where $\mathrm{OBC}$ is the partially-ordered "conglomerate" of iso-closed object classes in $\mathcal{C}$. We remark that (in a more restricted context) the functor $D$ and its left adjoint were considered previously in [T1], under the name Salbany correspondence; when translated into the language of closure operators [DT], the left adjoint of $D$ assigns to a class $\mathcal{B}$ precisely the $\mathcal{B}$-regular closure operator. Also a restriction of the functor $C$ appeared previously in the literature: in $[\mathrm{CHK}]$ it is used to establish the well-known correspondence between factorization systems and reflective subcategories, a generalization of which is described in [JT].

2.5 Our paradigmatic example is the category $\mathcal{C}=$ Top of topological spaces and the class $\mathcal{F}=\mathrm{Cl}$ of closed continuous maps. Then $c(\mathrm{Cl})$ is the class of proper or stably-closed maps in the sense of [B] (i.e., of those $f: X \rightarrow Y$ for which $f \times 1_{Z}$ is closed for all $Z$ ); general topologists usually call them perfect and define them as closed maps with compact fibres, although this characterization is not a good starting point for a categorical theory. We reserve the name perfect for the maps in $c(\mathrm{Cl}) \cap d(\mathrm{Cl})$; here $d(\mathrm{Cl})$ is the class of separated maps as in [Js] (i.e., those $f: X \rightarrow Y$ for which any $x \neq y$ with $f(x)=f(y)$ may be separated by disjoint neighbourhoods in $X$ ). Of course, $D(\mathrm{Cl})$ is the class of Hausdorff spaces, and $C(\mathrm{Cl})$ the class of compact spaces, thanks to the Kuratowski-Mrowka Theorem. (Note that we use "compact" in the usual topological sense, defined by the open-cover property, without any separation condition.) Hence, we use "compact" also for the maps in $c(\mathrm{Cl})$.

There is another equally important class in Top, namely, the class $\mathrm{Op}=c(\mathrm{Op})$ of open continuous maps. Here $C(\mathrm{Op})=$ Top, and $D(\mathrm{Op})$ is the class of discrete spaces. Less trivially, $d(\mathrm{Op})$ is the class of locally injective maps (every point of the domain has a neighbourhood on which the map maps injectively), while $\mathrm{Op} \cap d(\mathrm{Op})$ is the class of local homeomorphisms.

Next we provide a general reason why $\mathrm{Cl}$ and $\mathrm{Op}$ should play a distinguished role in Top; these are classes of "homomorphisms" definable in a much more general context!

2.6 (Closed and Open Morphisms with Respect to a Closure Operator) Let $(\mathcal{E}, \mathcal{M})$ be a proper, stable factorization system in $\mathcal{C}$. By $\operatorname{sub} X$ we denote the preordered class $\mathcal{M} / X$, and $f(m)$ denotes the $\mathcal{M}$-part of an $(\mathcal{E}, \mathcal{M})$-factorization of $m \cdot f, m \in \mathcal{M}$. A closure operator $c=$ $\left(c_{X}\right)_{X \in \mathcal{C}}$ (cf. [DG1], [DT]) is a family of extensive and monotone maps $c_{X}: \operatorname{sub} X \rightarrow \operatorname{sub} X$ which satisfy, for every $f: X \rightarrow Y$, the following equivalent conditions:

(i) $f\left(c_{X}(m)\right) \leqslant c_{Y}(f(m))$ for all $m \in \operatorname{sub}(X)$,

(ii) $c_{X}\left(f^{*}(n)\right) \leqslant f^{*}\left(c_{Y}(n)\right)$ for all $n \in \operatorname{sub}(Y)$,

(iii) $c_{X}(m) \leqslant f^{*}\left(c_{Y}(f(m))\right)$ for all $m \in \operatorname{sub}(X)$,

(iv) $f\left(c_{X}\left(f^{*}(n)\right)\right) \leqslant n$ for all $n \in \operatorname{sub}(Y)$.

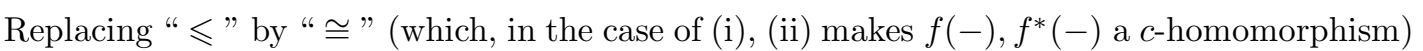
we obtain the notions of $f$ being (i) c-closed, (ii) c-open, (iii) c-initial, (iv) $c$-final; this defines the classes $\mathrm{Cl}(c), \operatorname{Op}(c), \operatorname{Ini}(c)$, Fin $(c)$, respectively. Each of these classes is closed under composition and contains all isomorphisms. The interelationships between them are discussed in [GT], [CGT2]; we mention in particular:

Pullback Ascent and Descent Theorem. Consider the pullback diagram 


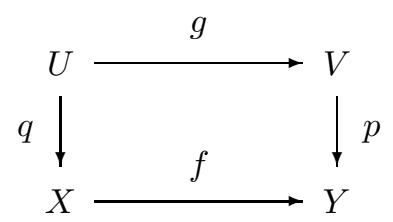

and the following two assertions:

(i) $f$ is c-closed (c-open, c-initial, c-final, respectively);

(ii) $g$ is c-closed (c-open, c-initial, c-final, respectively);

Then (i) $\Rightarrow$ (ii) if $q$ is c-initial, and (ii) $\Rightarrow$ (i) if $p$ is $c$-final.

Without additional condition, neither implication holds in general.

When can we expect at least $\mathrm{Cl}(c) \cap \operatorname{RegMono} \mathcal{C}$ and $\mathrm{Op}(c) \cap \operatorname{RegMono} \mathcal{C}$ to be stable under pullback? First of all, it is important to distinguish the notion of $c$-closed morphism $(=c$ preserving morphism in [CGT1] and earlier papers) from the notion of $c$-closed subobject: $m \cong c_{X}(m)$. Likewise, the notion of $c$-open morphism must be distinguished from the notion of $c$-open subobject $m$ of $X$, as given in [GT]: $m \wedge c_{X}(x) \leqslant c_{X}(m \wedge x)$ for all $x \in \operatorname{sub} X$. Despite the dissimilarity in definition, there is a remarkable symmetry in behaviour, as expressed by the following (slightly tricky):

Lemma. Let $m: M \rightarrow X$ be a morphism in $\mathcal{M}$. Then $m$ is c-closed (c-open) as a morphism if and only if $m$ is c-initial and c-closed (c-open) as a subobject of $X$.

The operator $c$ is hereditary if $\mathcal{M} \subseteq \operatorname{Ini}(c)$; hence, in this case there is no need to distinguish between the morphism and the subobject notions. In fact, for $c$-closedness this is true already when $c$ is weakly hereditary (i.e., when every subobject is $c$-dense in its $c$-closure), see [CGT1]. Since $c$-closed subobjects are always stable under pullback, also the morphism class $\mathrm{Cl}(c) \cap$ RegMonoC has this property when $c$ is weakly hereditary. Unfortunately, we cannot

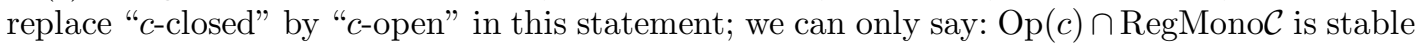
under pullback if the class of $c$-open subobjects has this property and $c$ is hereditary.

Objects and morphisms in $D(\mathrm{Cl}(c)), d(\mathrm{Cl}(c))$ are called $c$-separated, and those in $C(\mathrm{Cl}(c))$, $c(\mathrm{Cl}(c)) c$-compact. In particular, the papers [DG2], [CGT1] exhibit many examples; the reader is, however, alerted to the fact that in general, the notion of $c$-separation used in these papers may not coincide with the one given here when $c$ fails to be weakly hereditary: see 3.10.

2.7 (Summands in "Lextensive" Categories) As observed recently in [CPR], the finitely complete category $\mathcal{C}$ has binary sums (=coproducts) if and only if the sum $1+1$ exists in $\mathcal{C}$ and the functor "pulling back along injections"

$$
\mathrm{pb}_{1,1}: \mathcal{C} / 1+1 \rightarrow \mathcal{C} \times \mathcal{C}(\cong \mathcal{C} / 1 \times \mathcal{C} / 1)
$$

has a left adjoint; in this case, for every pair of objects $X, Y$,

$$
\operatorname{pb}_{X, Y}: \mathcal{C} / X+Y \rightarrow \mathcal{C} / X \times \mathcal{C} / Y
$$

has a left adjoint, given by sum. Let SumC be the class of all coproduct injections; it is closed under composition and contains all isomorphisms. Furthermore, if all functors $\mathrm{pb}_{X, Y}$ are full and faithful, then SumC is stable under pullback; this is true particularly when $\mathcal{C}$ is extensive (cf. [CLW], [CJ]), i.e., when $\mathrm{pb}_{1,1}$ (and therefore every $\mathrm{pb}_{X, Y}$ - see $\left.[\mathrm{CPR}]\right)$ ) is an equivalence of categories. But also without this hypothesis it makes sense to call a morphism $f$ decidable or (separable) if $\delta_{f}$ is a stable coproduct injection. For lextensive $\mathcal{C}$, the classes $D(\operatorname{SumC})$ and $d(\mathrm{SumC})$ have been studied in $[\mathrm{CJ}]$, but many of their properties remain valid without the hypothesis of extensivity, as we shall see in the next section. A prominent example of [CJ] is the dual of the category commutative rings, where a morphism $f: A \rightarrow B$ is separable if and 
only if $A$ is a separable $B$-algebra. Another example of $[\mathrm{CJ}]$ is $\mathcal{C}=$ Top where, in the notation of 2.5 , one has $D(\mathrm{SumC})=D(\mathrm{Op})$ and $d(\mathrm{SumC})=d(\mathrm{Cl} \cap \mathrm{Op})=d(\mathrm{Cl}) \cap d(\mathrm{Op})$.

In general, $C(\mathrm{SumC})$ is the class of universal summands of 1 ; hence, $C(\mathrm{SumC} / B)$ is the class of universal summands of $B$. Of course, here "universal" is redundant if $\mathcal{C}$ (and therefore $\mathcal{C} / B)$ is extensive.

2.8 (Cartesian Morphisms w.r.t. a Pointed Endofunctor) Let $T$ be an endofunctor of $\mathcal{C}$, "pointed" by a natural transformation $\eta: 1_{\mathcal{C}} \rightarrow T$, with $T 1 \cong 1$. The class Car $T$ of all morphisms for which the $\eta$-naturality diagram is a pullback diagram includes all isomorphisms and is closed under composition. These morphisms are called T-cartesian or trivialT-coverings in [JT], where those $T$ have been characterized for which $(\operatorname{Ver} T$, CarT) forms a factorization system, with $\operatorname{Ver} T$ the class of $T$-vertical morphisms (i.e., of those $f: X \rightarrow Y$ with $\eta_{Y}^{*}(T f) \cong$ $\left.1_{X}\right)$. They include the semi-left exact or admissible reflections of [CHK], [J], [CJKP], and the direct reflections of $[\mathrm{BG}]$, [Ho]. A particular consequence of those conditions is that CarT is stable under pullback. But also without this property it makes sense to consider the classes $D(\operatorname{Car} T)$ and $d(\operatorname{Car} T)$ of $T$-separable objects and morphisms in $\mathcal{C}$. The papers referred to above contain many examples, from both algebra and topology.

It is not difficult to prove that if every morphism $\eta_{X}$ is a universal regular epimorphism, then $C(\operatorname{Car} T)$ contains exactly those objects $X$ for which $T(X \times Y) \cong X \times T Y$ (canonically, for all objects $Y$ ). If $\operatorname{Car} T$ is stable under pullback, then $C(\operatorname{Car} T)=\operatorname{Fix} T=\left\{X \mid \eta_{X}\right.$ iso $\}$.

\section{A General Finite Theory of Separation and Compactness}

3.1 Following our leading example (see 2.5, 2.6), we call objects and morphisms in $D(\mathcal{F})$, $d(\mathcal{F}) \mathcal{F}$-separated, and those in $C(\mathcal{F}), c(\mathcal{F}) \mathcal{F}$-compact. We now derive some basic but useful properties for them, under the sole hypothesis that our morphism class $\mathcal{F} \subseteq$ MorC satisfies the following axiom:

$(A 1) \mathcal{F}$ is closed under composition and contains all isomorphisms.

Recall from 2.1, 2.2 that then $d(\mathcal{F})$ and $c(\mathcal{F})$ are both closed under composition and stable under pullback, and that $d(\mathcal{F})$ is left-cancellable, while $c(\mathcal{F})$ is left-cancellable with respect to monomorphisms.

3.2 Lemma. An object $X$ is $\mathcal{F}$-separated if and only if for every equalizer diagram

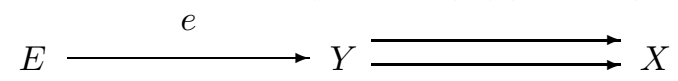

e lies in $\mathcal{F}$ (or, equivalently, in $c(\mathcal{F})$ ).

Proof. Such equalizers are precisely the pullbacks of $\delta_{X}: X \rightarrow X \times X$.

3.3 Proposition. In each (1) and (2), the three given conditions for an object $X$ are equivalent:

(1) (i) $X$ is $\mathcal{F}$-separated;

(ii) every morphism with domain $X$ is $\mathcal{F}$-separated;

(iii) there is an $\mathcal{F}$-separated morphism with domain $X$ and $\mathcal{F}$-separated codomain.

(2) (i) $X$ is $\mathcal{F}$-compact;

(ii) every morphism with domain $X$ and $\mathcal{F}$-separated codomain is $\mathcal{F}$-compact;

(iii) there is an $\mathcal{F}$-compact morphism with domain $X$ and $\mathcal{F}$-compact codomain.

Proof. For a morphism $f: X \rightarrow Y$, consider the commutative diagram 


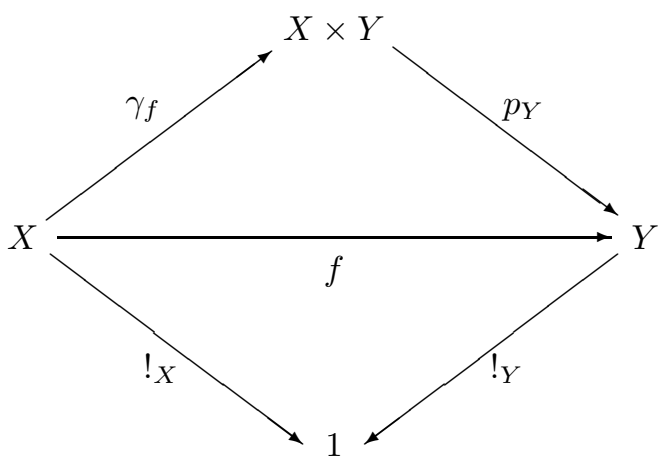

with $\gamma_{f}=<1_{X}, f>\cong\left(f \times 1_{Y}\right)^{*}\left(\delta_{X}\right)$ the graph of $f$. Since $c(\mathcal{F})$ is closed under composition, the diagram shows (i) $\Rightarrow$ (ii) and (iii) $\Rightarrow$ (i) of (2), while (ii) $\Rightarrow$ (iii) is trivial (take $f=!_{X}$ ). For (1) it suffices to look just at the lower triangle of $(3.2)$, since $d(\mathcal{F})$ is not only closed under composition but also left-cancellable.

Remark. Since a compact subspace of a non-Hausdorff space need not be closed, we see in particular that in general $c(\mathcal{F})$ is not left-cancellable. However, the statement that $c(\mathcal{F})$ is left-cancellable with respect to monomorphisms may be strengthened to statement (2) below.

\subsection{Corollary.}

(1) A morphism $f: X \rightarrow Y$ with $Y \mathcal{F}$-separated $(\mathcal{F}$-separated and $\mathcal{F}$-compact) is $\mathcal{F}$-separated $(\mathcal{F}$-compact) if and only if $X$ is $\mathcal{F}$-separated $(\mathcal{F}$-compact $)$.

(2) If $g \cdot f$ is $\mathcal{F}$-compact with $g \mathcal{F}$-separated, then also $f$ is $\mathcal{F}$-compact.

(3) If $f$ is $\mathcal{F}$-compact and $g$ has $\mathcal{F}$-compact domain, then also $f^{*}(g)$ has $\mathcal{F}$-compact domain. In particular, fibres of $\mathcal{F}$-compact morphisms are $\mathcal{F}$-compact.

Proof. (2) Apply 3.3(2)(i) $\Rightarrow$ (ii) to $\mathcal{C}^{B}$ and $\mathcal{F}^{B}$ (see 2.3), with $B=\operatorname{codomain}(g)$.

(3) Apply $3.3(2)$ (iii) $\Rightarrow$ (i) to $g^{*}(f)$ (rather than to $f$ ), and consider in particular the case $\operatorname{domain}(g)=1$.

Remark. A morphism $f$ in $\mathcal{F}$ with the property that $f^{*}(g)$ has $\mathcal{F}$-compact domain for every $g$ with $\mathcal{F}$-compact domain need not be $\mathcal{F}$-compact: consider $\mathcal{C}=$ Top $/ B$ with $B$ a two-point indiscrete space and $\mathcal{F}$ the class of closed maps (see [CGT], 5.14). In particular, morphisms in $\mathcal{F}$ with $\mathcal{F}$-compact fibres need not be $\mathcal{F}$-compact.

3.5 Corollary. $D(\mathcal{F})$ is closed under monomorphisms, and $C(\mathcal{F})$ is closed under morphisms in $c(\mathcal{F})$.

Proof. Every monomorphism is $\mathcal{F}$-separated; hence, one may invoke $3.3(1)($ iii $) \Rightarrow(\mathrm{i})$. The second assertion rephrases $3.3(2)(\mathrm{iii}) \Rightarrow(\mathrm{i})$.

3.6 Proposition. $C(\mathcal{F})$ is closed under finite products, and $D(\mathcal{F})$ and $C D(\mathcal{F})=C(\mathcal{F}) \cap$ $D(\mathcal{F})$ are closed under finite limits in $\mathcal{C}$.

Proof. Closure of $C(\mathcal{F})$ and $D(\mathcal{F})$ under binary products follows from

$$
!_{X \times Y}=!_{Y} \cdot p_{Y} \text { and } \delta_{X \times Y} \cong \delta_{X} \times \delta_{Y}=\left(1_{X \times X} \times \delta_{Y}\right)\left(\delta_{X} \times 1_{Y}\right),
$$

and trivially $1 \in C D(\mathcal{F})$. Furthermore, for the equalizer diagram (3.1), one has $e \in c(\mathcal{F})$ when $X \in D(\mathcal{F})$, hence $E \in C(\mathcal{F})$ when $Y \in C(\mathcal{F})$, by 3.5. This shows closure of $C D(\mathcal{F})$ under equalizers. The same holds true for $D(\mathcal{F})$, again thanks to 3.5 . 
Remark. For the sake of completeness, we mention also the following properties:

(1) if $X \times Y \in D(\mathcal{F})$ with $\mathcal{C}(1, Y) \neq \emptyset$, then $X \in D(\mathcal{F})$;

(2) if $X \times Y \in C(\mathcal{F}), Y \in D(\mathcal{F})$ with $\mathcal{C}(1, Y) \neq \emptyset$, then $X \in C(\mathcal{F})$.

In fact, the hypothesis of $(2)$ gives $p_{Y} \in c(\mathcal{F})$ with $3.3(2)(\mathrm{i}) \Rightarrow($ ii), and for every $y: 1 \rightarrow Y$ one has $!_{X} \cong y^{*}\left(p_{Y}\right)$, hence $X \in C(\mathcal{F})$. This proves $(2)$, and for (1) one proceeds analogously.

3.7 Proposition. Let $\mathcal{E}$ be a pullback-stable class of morphisms with the property that $\mathcal{F}$ is right-cancellable w.r.t. $\mathcal{E}$ (so that $g \cdot f \in \mathcal{F}$ and $f \in \mathcal{E}$ imply $g \in \mathcal{F}$ ). Then, for $f: X \rightarrow Y$ in $\mathcal{E}$ one has:

(1) $X \mathcal{F}$-compact implies $Y \mathcal{F}$-compact;

(2) $Y \mathcal{F}$-separated and f $\mathcal{F}$-compact imply $X \mathcal{F}$-separated.

Proof. (1) Stability of $\mathcal{E}$ and the cancellation property of pullbacks show that with $\mathcal{F}$ also $c(\mathcal{F})$ is right-cancellable w.r.t. $\mathcal{E}$. Hence, $!_{X}=!_{X} \cdot f \in c(\mathcal{F})$ implies $!_{Y} \in c(\mathcal{F})$.

(2) With $f \in c(\mathcal{F})$ also $f \times f=\left(1_{Y} \times f\right)\left(f \times 1_{X}\right) \in c(\mathcal{F})$. Hence, $\delta_{Y} \times f=(f \times f) \cdot \delta_{X} \in c(\mathcal{F})$ implies $\delta_{Y} \in c(\mathcal{F})$.

Remarks. (1) In Top, with $\mathcal{F}=\mathrm{Cl}$ or $\mathcal{F}=$ Op, we may choose for $\mathcal{E}$ the class of epimorphisms. More generally, in the setting of 2.6 , with $\mathcal{F}=\mathrm{Cl}(c)$ or $\mathcal{F}=\mathrm{Op}(c), \mathcal{E}$ satisfies the hypothesis of 3.7 .

(2) We note that in $3.7(2)$ we only use that $c(\mathcal{F})$ is right-cancellable w.r.t. $c(\mathcal{F}) \cap \mathcal{E}$. This property holds also in the setting of 2.8 when we take for $\mathcal{E}$ those morphisms $f$ for which $T f$ is a descent morphism (i.e., a universal regular epimorphism).

3.8 In order to discuss closedness of $C(\mathcal{F})$ and $D(\mathcal{F})$ under finite sums, we assume $\mathcal{C}$ to be extensive (see 2.7) and prove:

Theorem. Let $\mathcal{C}$ be extensive and $\mathcal{F}$ be closed under finite sums (so that with $f_{i}: X_{i} \rightarrow Y_{i}$ also $f_{1}+f_{2}$ lies in $\left.\mathcal{F}\right)$. Then also $c(\mathcal{F})$ and $d(\mathcal{F})$ are closed under finite sums. Furthermore, $C(\mathcal{F})$ and $D(\mathcal{F})$ are closed under finite sums if and only if $1+1 \in C(\mathcal{F})$ and $1+1 \in D(\mathcal{F})$, respectively.

Proof. Extensivity gives the formulas

$$
\begin{gathered}
\delta_{f_{1}+f_{2}} \cong \delta_{f_{1}}+\delta_{f_{2}}, \\
h^{*}\left(f_{1}+f_{2}\right) \cong h_{1}^{*}\left(f_{1}\right)+h_{2}^{*}\left(f_{2}\right),
\end{gathered}
$$

with $h: Z \rightarrow Y_{1}+Y_{2}, h_{i} \cong j_{i}^{*}(h)$, and $j_{i}: Y_{i} \rightarrow Y_{1}+Y_{2}$ a coproduct injection. These show closedness of $c(\mathcal{F})$ and $d(\mathcal{F})$ under finite coproducts. Since

$$
!_{X+Y} \cong !_{2} \cdot\left(!_{X}+!_{Y}\right)
$$

with $2 \cong 1+1$, the corresponding statement for $C(X)$ follows immediately. For $D(X)$, one uses the formula (observed in [CJ])

$$
\delta_{X+Y} \cong k \cdot\left(\delta_{X}+\delta_{Y}\right),
$$

with $k=<\pi_{1}^{X}+\pi_{1}^{Y}, \pi_{2}^{X}+\pi_{2}^{Y}>:(X \times X)+(Y \times Y) \rightarrow(X+Y) \times(X+Y)$. By extensitivity, $\left(\pi_{1}^{X}+\right.$ $\left.\pi_{1}^{Y}, \pi_{2}^{X}+\pi_{2}^{Y}\right)$ is the kernelpair of $!_{X}+!_{Y}$, so that $k$ is the equalizer of $\left(!_{X}+!_{Y}\right) \pi_{1},\left(!_{X}+!_{Y}\right) \pi_{2}$. Hence, if $1+1 \in D(\mathcal{F})$, with 3.2 one obtains $k \in c(\mathcal{F})$.

Remarks. (1) The assertions concerning $d(\mathcal{F})$ and $D(\mathcal{F})$ require closedness under finite sums only for $\mathcal{F} \cap \operatorname{RegMono} \mathcal{C}$, not for the whole class $\mathcal{F}$.

(2) The trivial example $\mathcal{F}=$ Iso $\mathcal{C}$ shows that the conditions $1+1 \in C(\mathcal{F})$ and $1+1 \in D(\mathcal{F})$ do not come for free, even when $\mathcal{F}$ (and therefore $c(\mathcal{F})$ and $d(\mathcal{F})$ ) are closed under finite sums. 
3.9 In general, neither $D(\mathcal{F})$ nor $C(\mathcal{F})$ is closed under infinite products in $\mathcal{C}$. For example, in Top, failure of a countable product of discrete spaces to be discrete confirms this claim not only for $D(\mathrm{Op})$ but also for $C(\mathcal{F})$, with $\mathcal{F}$ the class of maps which preserve $b$-closed sets; here $M \subseteq X$ is $b$-closed if for every $x \in X \backslash M$ there is a neighbourhood $U$ with $U \cap M \cap \overline{\{x\}} \neq \emptyset$.

However, if $\mathcal{F} \cap \operatorname{RegMonoC}$ is closed under intersection (multiple pullback), then $D(\mathcal{F})$ and $d(\mathcal{F})$ are closed under (infinite) direct products, and $d(\mathcal{F})$ is closed under infinite fibred products (=mutiple pullback). To wit, consider $g_{i}: X_{i} \rightarrow Y_{i}$ and $g=\prod_{i \in I} g_{i}: \prod_{i \in I} X_{i} \rightarrow$ $\prod_{i \in I} Y_{i}$, and for $f_{i}: X_{i} \rightarrow Y$, let $f=\bigwedge_{i \in I} f_{i}: X \rightarrow Y$ be the fibred product. Then the formulas

$$
\begin{aligned}
& \left.h^{*}(f) \cong \bigwedge_{i} h^{*}\left(f_{i}\right) \text { (for every } h \text { with codomain } Y\right), \\
& \left.\delta_{f} \cong\left(p_{i} \times p_{i}\right)^{*}\left(\delta_{f_{i}}\right) \text { (with projections } p_{i}: X \rightarrow X_{i}\right), \\
& \left.g \cong \bigwedge_{i} \pi_{i}^{*}\left(g_{i}\right) \text { (with projections } \pi_{i}: \prod_{j} Y_{j} \rightarrow Y_{i}\right), \\
& \delta_{g} \cong \prod_{i} \delta_{g_{i}}, \\
& \text { show }
\end{aligned}
$$

(1) $f_{i} \in d(\mathcal{F})$ for all $i \in I$ implies $\bigwedge_{i} f_{i} \in d(\mathcal{F})$,

(2) $g_{i} \in d(\mathcal{F})$ for all $i \in I$ implies $\prod_{i} g_{i} \in d(\mathcal{F})$,

(3) $X_{i} \in D(\mathcal{F})$ for all $i \in I$ implies $\prod_{i} X_{i} \in D(\mathcal{F})$.

Note that for $\mathcal{F}=\mathrm{Cl}$ in Top, the assumption that $\mathcal{F} \cap$ RegMonoC be closed under intersection is trivially satisfied. However, without restriction to regular monomorphisms things become more complicated, even in our guiding example where closure of $C(\mathcal{F})$ under direct products is precisely Tychonoff's Theorem, and where closure of $c(\mathcal{F})$ under direct products is the assertion of the Frolik-Bourbaki Theorem. We recall here only two strategies which have been used in [CT], [CGT1] to prove generalizations of these theorems in the context of 2.6.

First, consider an infinite product $X_{I}=\prod_{i} X_{i}$ as an inverse limit of finite products:

$$
X_{I} \cong \lim _{\longleftarrow} X_{F} \quad(F \subseteq I \text { finite }) .
$$

Then also $X_{I} \times Y \cong \lim _{F}\left(X_{F} \times Y\right)$ for all objects $Y$, and the projection $q_{I}: X_{I} \times Y \rightarrow Y$ is an inverse limit of the projections $q_{F}: X_{F} \times Y \rightarrow Y$. Hence, if $q_{F} \in \mathcal{F}$ for all $F \subseteq I$ finite implies $q_{I} \in \mathcal{F}$, then

(4) $X_{i} \in C(\mathcal{F})$ for all $i \in I$ implies $\prod_{i} X_{i} \in C(\mathcal{F})$.

Furthermore, if the hypothesis of (4) is satisfied not only for $\mathcal{C}$ but for every slice $\mathcal{C} / B$ (and for $\mathcal{F}^{B}$ in lieu of $\mathcal{F}$ ), then $(1),(2)$ hold true for $c(\mathcal{F})$ instead of $d(\mathcal{F})$.

Remark. Of course, in general, $D(\mathcal{F})$ and $C(\mathcal{F})$ are not closed under infinite coproducts either, but sufficient conditions could be established as in 3.8, with an infinite version of the extensity axiom.

3.10 Of the many examples in the context of 2.6, we mention here only the following: take for $c$ the so-called Theta-closure in Top; hence, for $M \subseteq X$ let $\theta_{X}(M)$ be the set of those $x \in X$ for which each closed neighbourhood meets $M$. A space $X$ is $\theta$-compact precisely when it is $H$-closed, i.e., when it is closed in every Hausdorff extension space. It was shown in [CGT1] that the techniques of 3.9 give the Chevalley-Frink product theorem: $C(\mathrm{Cl}(\theta))$ is stable under direct products in Top.

Since $\theta$ fails to be weakly hereditary, a $\theta$-closed subobject $E \subseteq Y$ may not give a $\theta$-closed map $E \hookrightarrow Y$. Consequently, the notions of $\theta$-Hausdorffness as used in [CGT1] and earlier papers and of $\theta$-separation as used in this paper may differ; more precisely, an object $X$ with $\theta$-closed diagonal $\Delta_{X} \subseteq X \times X$ may fail to be $\theta$-separated. While the former property precisely means that $X$ is an Urysohn space (distinct points may be separated by disjoint closed neighbourhoods), $\theta$-separatedness of $X$ entails the additional property that (in the language of 
[DT]) $\theta$ is hereditary with respect to $\{X\}$-closed subsets; this means that for all $f, g: Y \rightarrow X$, the $\theta$-closure of every $N \subseteq E=\{y \in Y \mid f(y)=g(y)\}$ in $E$ can be computed in $Y$. Although $E$ is a $\theta$-closed subspace of $Y$, this additional property does not come for free:

Example (M.M. Clementino; see also [DT]) Obtain the space $Y$ by providing the unit interval with the coarsest topology that is finer than the Euclidean topology and in which $F=\left\{\frac{1}{n} \mid n \in\right.$ $\mathbf{N}\}$ is closed. Then $Y$ is an Urysohn space, and $E=F \cup\{0\}$ is $\theta$-closed in $Y$, hence it is the equalizer of two maps $Y \Longrightarrow X$, with $X$ an Urysohn space (see [DT]). However, since $\theta_{E}(F)=F$ but $\theta_{Y}(F)=E$, the condition of 3.2 is violated. Consequently, $X$ fails to be $\theta$-separated.

\section{Embeddable and Absolute Objects and Morphisms}

4.1 We continue to work with a class $\mathcal{F}$ of morphism satisfying the condition $(A 1)$. In addition, we fix a left-cancellable classs $\mathcal{M}$ with $\operatorname{RegMonoC} \subseteq \mathcal{M} \subseteq$ MonoC which is closed under composition and stable under pullback. We may then define:

$$
\begin{gathered}
T_{\mathcal{M}}(\mathcal{F})=\{X \mid \exists m: X \rightarrow K \text { in } \mathcal{M}: K \in C D(\mathcal{F})\} \\
H_{\mathcal{M}}(\mathcal{F})=\{X \mid \forall m: X \rightarrow K \text { in } \mathcal{M}:(K \in D(\mathcal{F})) \Longrightarrow m \in c(\mathcal{F})\} .
\end{gathered}
$$

In our paradigmatic example, with $\mathcal{M}=\operatorname{RegMonoTop}, T_{\mathcal{M}}(\mathrm{Cl})$ is the class of Tychonoff spaces, and $H_{\mathcal{M}}(\mathrm{Cl})$ is the class of $H$-closed spaces (see 3.10). In general, we call the objects

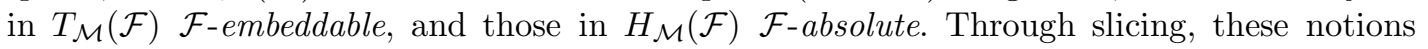
extend to morphisms; hence, one defines morphism classes $t_{\mathcal{M}}(\mathcal{F})$ and $h_{\mathcal{M}}(\mathcal{F})$, as follows: for $f: X \rightarrow Y$ in $\mathcal{C}$

$$
\begin{gathered}
f \in t_{\mathcal{M}}(\mathcal{F}) \Leftrightarrow(X, f) \in T_{\mathcal{M}^{Y}}\left(\mathcal{F}^{Y}\right) \Leftrightarrow \exists m \in \mathcal{M}, k \in c d(\mathcal{F}): f=k \cdot m, \\
f \in h_{\mathcal{M}}(\mathcal{F}) \Leftrightarrow(X, f) \in H_{\mathcal{M}^{Y}}\left(\mathcal{F}^{Y}\right) \Leftrightarrow \forall m \in \mathcal{M}, k \in d(\mathcal{F}):(f=k \cdot m \Rightarrow m \in c(\mathcal{F})) .
\end{gathered}
$$

4.2 Proposition. In each (1) and (2), the three given conditions for an object $X$ are equivalent:

(1) (i) $X$ is $\mathcal{F}$-embeddable;

(ii) every morphism with domain $X$ is $\mathcal{F}$-embeddable;

(iii) there is an $\mathcal{F}$-embeddable morphism with domain $X$ and $\mathcal{F}$-compact, $\mathcal{F}$-separated codomain.

(2) (i) $X$ is $\mathcal{F}$-absolute;

(ii) every morphism with domain $X$ and $\mathcal{F}$-separated codomain is $\mathcal{F}$-absolute;

(iii) there is an $\mathcal{F}$-absolute morphism with domain $X$ and $\mathcal{F}$-compact codomain.

Proof. For morphisms $f: X \rightarrow Y$ and $m: X \rightarrow Z$, consider the diagram

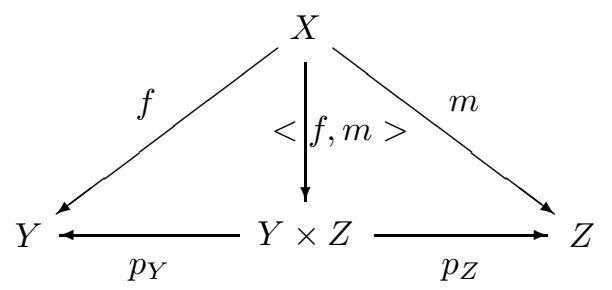

If $Z \in C D(\mathcal{F})$, then $p_{Y} \cong p_{Z}^{*}\left(!_{Z}\right) \in c d(\mathcal{F})$, and $m=p_{Z} \cdot\langle f, m>\in \mathcal{M}$ implies $\langle f, m>\in \mathcal{M}$; this proves $(1)(\mathrm{i}) \Rightarrow($ ii). Similarly, for $(2)($ iii) $\Rightarrow(\mathrm{i})$, the hypotheses $m \in \mathcal{M}, Z \in D(\mathcal{F})$ give 
$<f, m>\in \mathcal{M}, \quad p_{Y} \in d(\mathcal{F})$, so that $f \in h_{\mathcal{M}}(\mathcal{F})$ gives $\langle f, m>\in c(\mathcal{F})$; furthermore, $Y \in C(\mathcal{F})$ implies $p_{Z} \in c(\mathcal{F})$, hence $m=p_{Z} \cdot\langle f, m>\in c(\mathcal{F})$.

Let us now assume $f=k \cdot m$ with $m \in \mathcal{M}$. If $k \in c d(\mathcal{F})$ and $Y \in C D(\mathcal{F})$ we obtain $Z \in C D(\mathcal{F})$ with 3.3 ; this proves (1)(iii) $\Rightarrow(\mathrm{i})$. For $(2)(\mathrm{i}) \Rightarrow(\mathrm{ii})$, assume $k \in d(\mathcal{F})$ and $Y \in D(\mathcal{F})$, hence $Z \in D(\mathcal{F})$; if $X \in H_{\mathcal{M}}(\mathcal{F})$, this gives $m \in c(\mathcal{F})$, as desired.

The implications (ii) $\Rightarrow$ (iii) are trivial in both cases.

\subsection{Corollary.}

(1) $t_{\mathcal{M}}(\mathcal{F})$ is left-cancellable and stable under pullback; furthermore, if $f \in t_{\mathcal{M}}(\mathcal{F})$ and $g \in$ $\operatorname{cd}(\mathcal{F})$, then $g \cdot f \in t_{\mathcal{M}}(\mathcal{F})$.

(2) If $g \cdot f \in h_{\mathcal{M}}(\mathcal{F})$ with $g \in d(\mathcal{F})$, then also $f \in h_{\mathcal{M}}(\mathcal{F})$; if $f \in h_{\mathcal{M}}(\mathcal{F})$ and $g \in c(\mathcal{F})$, then $g \cdot f \in h_{\mathcal{M}}(\mathcal{F})$.

Proof. These assertions follow from the sliced version of 4.2 and the definition 4.1.

4.4 Proposition. $T_{\mathcal{M}}(\mathcal{F})$ is closed under finite limits and $\mathcal{M}$-subobjects.

Proof. Trivial, since $\mathcal{M}$ is closed under finite direct products and under composition, and since RegMonoC $\subseteq \mathcal{M}$.

Remark. Similarly to 3.6 one has:

(1) if $X \times Y \in T_{\mathcal{M}}(\mathcal{F})$ with $\mathcal{C}(1, Y) \neq \emptyset$, then $X \in T_{\mathcal{M}}(\mathcal{F})$;

(2) if $X \times Y \in H_{\mathcal{M}}(\mathcal{F})$ with $Y \in D(\mathcal{F})$ and $\mathcal{C}(1, Y) \neq \emptyset$, then $X \in H_{\mathcal{M}}(\mathcal{F})$.

For the proof one proceeds as in 3.6, observing that for every $m: X \rightarrow K$ and $y: 1 \rightarrow Y$ one has $m \cong<1_{K}, y>^{*}\left(m \times 1_{Y}\right)$.

4.5 Theorem. In the "lattice" of subclasses of $\mathrm{ObC}$ one has the following diagram:

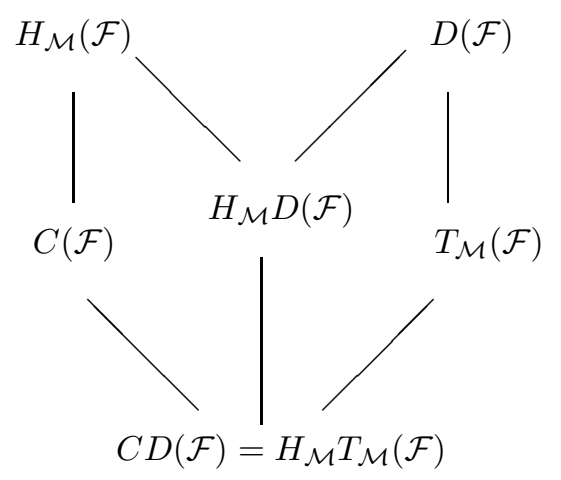

In particular, the intersection $H_{\mathcal{M}} T_{\mathcal{M}}(\mathcal{F})=H_{\mathcal{M}}(\mathcal{F}) \cap T_{\mathcal{M}}(\mathcal{F})$ does not depend on $\mathcal{M}$.

Proof. $C(\mathcal{F}) \subseteq H_{\mathcal{M}}(\mathcal{F})$ follows from 3.3(2), and $T_{\mathcal{M}}(\mathcal{F}) \subseteq D(\mathcal{F})$ from 3.5. Trivially, $C D(\mathcal{F}) \subseteq$ $T_{\mathcal{M}}(\mathcal{F})$, hence $C D(\mathcal{F}) \subseteq H_{\mathcal{M}} T_{\mathcal{M}}(\mathcal{F})$. Since for $X \in H_{\mathcal{M}} T_{\mathcal{M}}(\mathcal{F})$ one has $m: X \rightarrow K$ in $\mathcal{M}$ with $K \in C D(\mathcal{F})$ and then $m \in c d(\mathcal{F}), X \in C D(\mathcal{F})$ follows with 3.3.

4.6 Corollary. $h_{\mathcal{M}} t_{\mathcal{M}}(\mathcal{F})=c d(\mathcal{F})$. In particular, a morphism with $\mathcal{F}$-embeddable domain is $\mathcal{F}$-compact and $\mathcal{F}$-separated if and only if it is $\mathcal{F}$-absolute.

Proof. The first statement follows from the sliced version of 4.5, which implies also the second statement when combined with $4.2(1)(\mathrm{i}) \Rightarrow(\mathrm{ii})$. 
4.7 The last Corollary gives in particular the first part of the Isbell-Henriksen Theorem (cf. $[\mathrm{HI}])$ which says that for a continuous map $f: X \rightarrow Y$ of Tychonoff spaces the following conditions are equivalent: (i) $f$ is perfect; (ii) $f$ cannot be extended to a Hausdorff space of which $X$ is a proper dense subspace; (iii) the extension $\beta f: \beta X \rightarrow \beta Y$ of $f$ to the Stone-Čech compactifications maps $\beta X \backslash X$ into $\beta Y \backslash Y$. In categorical terms, (iii) means that

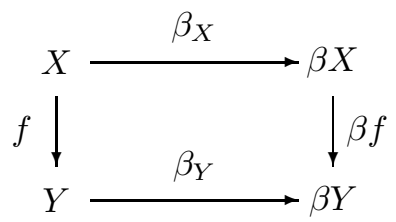

is a pullback diagram; i.e., $f$ is $\beta$-cartesian. In our general context we must assume the existence of the reflexions $\beta_{X}$, and that these are " $\mathcal{F}$-dense". More precisely, we now assume:

$(A 2) \quad$ Every $X \in T_{\mathcal{M}}(\mathcal{F})$ has a reflexion $\beta_{X}: X \rightarrow \beta X$ into $C D(\mathcal{F})$, and if $\beta_{X}=n \cdot m \cdot l \in \mathcal{M}$ with $n, m, l \in \mathcal{M}$ and $m \in c(\mathcal{F})$, then $m$ is an isomophism.

4.8 Theorem. The following conditions are equivalent for $f: X \rightarrow Y$ with $X, Y \in T_{\mathcal{M}}(\mathcal{F})$ :

(i) $f \in c(\mathcal{F})$;

(ii) if $f=(X \stackrel{m}{\rightarrow} Z \rightarrow Y)$ with $m \in \mathcal{M}, Z \in D(\mathcal{F})$, then $m \in c(\mathcal{F})$;

(iii) diagram (4.3) is a pullback diagram.

Proof. (i) $\Rightarrow$ (ii) Since with $Z \in D(\mathcal{F})$ also $(Z \rightarrow Y) \in d(\mathcal{F})$, we can just use $c(\mathcal{F}) \subseteq h_{\mathcal{M}}(\mathcal{F})$. (ii) $\Rightarrow$ (iii) Given diagram (4.3), form the pullback diagram

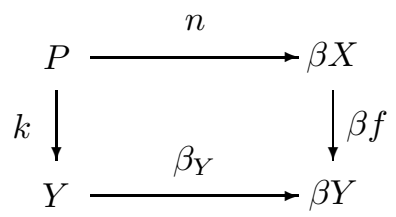

and consider the morphism $m: X \rightarrow P$ with $k \cdot m=f, n \cdot m=\beta_{X}$. First of all, since $X, Y \in T_{\mathcal{M}}(\mathcal{F})$, the reflexion property and left-cancellability of $\mathcal{M}$ give $\beta_{X}, \beta_{Y} \in \mathcal{M}$, hence also $m, n \in \mathcal{M}$ (with pullback stability). Since $P \in D(\mathcal{F})$, the hypothesis gives $m \in c(\mathcal{F})$, hence $m \in \operatorname{Iso}(\mathcal{C})$ with $(A 2)$. (iii) $\Rightarrow($ i) Since $\beta f \in c(\mathcal{F})$ by $3.3(2)$ (i) $\Rightarrow($ ii), also $f \in c(\mathcal{F})$.

Remark. The Theorem says in particular that, when restricted to the subcategory $T_{\mathcal{M}}(\mathcal{F})$, the class $c(\mathcal{F})=c d(\mathcal{F})$ is precisely the class $\operatorname{Car} \beta$ of $\beta$-cartesian morphisms. Next we shall see that the class $\operatorname{Ver} \beta$ of $\beta$-vertical morphisms is precisely the class $\beta^{-1}(\operatorname{Iso} C D(\mathcal{F}))$, and that $(\operatorname{Ver} \beta, \operatorname{Car} \beta)$ is an orthogonal factorization system.

4.9 Theorem (Antiperfect-Perfect Factorization). (1) Every morphism $f: X \rightarrow Y$ in $T_{\mathcal{M}}(\mathcal{F})$ can be factored as $f=k \cdot m$ with $k \quad \mathcal{F}$-perfect, $m: X \rightarrow P \in \mathcal{M}$ and $\beta m: \beta X \rightarrow \beta P$ an isomorphism.

(2) For every commutative diagram 


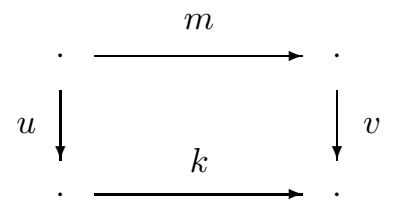

in $T_{\mathcal{M}}(\mathcal{F})$ with $k \quad \mathcal{F}$-perfect and $\beta m$ an isomorphism, there is exactly one morphism $w$ with $w \cdot m=u$ and $k \cdot w=v$.

Proof. According to Theorem 2.7 of [JT], it suffices to show that $m$ as constructed in the proof of 4.8 is $\beta$-vertical, and for that it suffices to show that

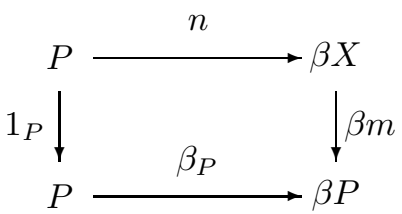

commutes. Let $e: E \rightarrow P$ be the equalizer of $\beta_{P}, \beta_{m} \cdot n$; since $\beta P \in D(\mathcal{F}), e \in c(\mathcal{F})$. With the morphism $l: X \rightarrow E$ with $e \cdot l=m$ we obtain $\beta_{X}=n \cdot e \cdot l$, so that $e \in$ IsoC follows with $(A 2)$. This completes the proof.

4.10 Although Theorem 4.8 (see $[\mathrm{CGT}]$ ) and 4.9 (see $[\mathrm{Ho}]$ ) are essentially known for $\mathcal{F}=$ $\mathrm{Cl}(c)$ and $c$ an idempotent, hereditary closure operator (see 2.6), little is known especially about the morphisms considered in this section, even in the case of our paradigmatic example $\mathcal{C}=$ Top, $\mathcal{F}=$ Cl. For example, one would expect that maps in $t_{\mathcal{M}}(\mathcal{F})$ (which are precisely restrictions of perfect maps) can be characterized in terms of $[0,1]$-valued maps. Dyckhoff $[\mathrm{Dy}]$, Pasynkov [P] and Künzi $[\mathrm{KP}]$ have studied intensively so-called Tychonoff maps; these are separated (see 2.5) maps $f: X \rightarrow Y$ with the property that for every closed set $F \subseteq X$ and every $x \in X \backslash F$ there is an open neighbourhood $U \subseteq Y$ of $f(x)$ and a (continuous) map $g: f^{-1}(U) \rightarrow[0,1]$ with $g(x)=0$ and $g\left(F \cap f^{-1}(U)\right) \subseteq\{1\}$. Since a space $X$ is Tychonoff if and only of $X \rightarrow 1$ is Tychonoff, and since every Tychonoff map is Cl-embeddable, one is tempted to believe that also the converse proposition is true. However, solving an open problem of $[\mathrm{P}]$, recently Zouboff $[\mathrm{Z}]$ exhibited an example of a perfect (hence, in particular Cl-embeddable) map which fails to be a Tychonoff map.

4.11. For a varietal theory $\mathcal{J}$ in the sense of Lawvere-Linton, Diers [Di] establishes an adjunction

$$
\operatorname{Set}(\mathcal{J}) \underset{\frac{A}{X}}{\frac{\perp}{X}} \operatorname{Alg}(\mathcal{J})^{o p}
$$

between the dual of (the) variety of $\mathcal{J}$-algebras and the topological category $\operatorname{Set}(\mathcal{J})$ of so-called $\mathcal{J}$-sets; as it induces an idempotent monad, it gives a duality between the fixed subcategories on both sides, given by functional $\mathcal{J}$-algebras and so-called algebraic $\mathcal{J}$-sets, respectively:

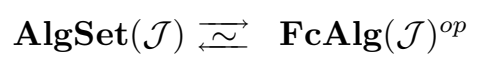

The functional $\mathcal{J}$-algebras are precisely the subalgebras of powers of the initial $\mathcal{J}$-algebra $K$. By definition, $\operatorname{AlgSet}(\mathcal{J})$ consists of those $\mathcal{J}$-sets $X$ for which the unit morphism $\eta_{X}: X \rightarrow$ 
$X(A(X))$ is an isomorphism; it is a subcategory of $\operatorname{SepSet}(\mathcal{J})$, which consists of the $\mathcal{J}$-sets $X$ for which $\eta_{X}$ is an embedding (=regular monomorphism).

It is shown in [Di] that the categories $\operatorname{SepSet}(\mathcal{J})$ and $\operatorname{AlgSet}(\mathcal{J})$ fit perfectly into the setting of this paper: one defines the Zariski closure $\zeta_{X}(M)$ of $M \subseteq X \in \operatorname{Set}(\mathcal{J})$ as the set of all $x \in X$ which satisfy

$$
\forall u, v \in A(X):\left(\left.u\right|_{M}=\left.v\right|_{M} \Rightarrow u(x)=v(x)\right) ;
$$

here $A(X)$ is the subalgebra of $K^{X}$ which gives the structure of the $\mathcal{J}$-set $X$. For this idempotent and weakly hereditary closure operator of $\operatorname{Set}(\mathcal{J})$ one obtains

$$
\operatorname{SepSet}(\mathcal{J})=D(\mathrm{Cl}(\zeta)), \quad \operatorname{AlgSet}(\mathcal{J})=H_{\mathcal{M}} D(\mathrm{Cl}(\zeta)),
$$

with $\mathcal{M}$ the class of embeddings.

\section{References}

B. N. Bourbaki, Topologie Générale, Ch. I et II, Third ed.(Paris, 1961).

BG. G. C. L. Brümmer and E. Giuli, Splitting operators, preprint (1997).

CJ. A. Carboni and G. Janelidze, Decidable (=separable) objects and morphisms in lextensive categories, J. Pure Appl. Algebra 110 (1996) 219-240.

CJKP. A. Carboni, G. Janelidze, G. M. Kelly and R. Paré, On localization and stabilization for factorization systems, Appl. Categorical Structures 5 (1997) 1-58.

CLW. A. Carboni, S. Lack and R. F. C. Walters, Introduction to extensive and distributive categories, J. Pure Appl. Algebra 84 (1993) 145-158.

CPR. A. Carboni, M. C. Pedicchio and J. Rosický, Syntactic characterizations of various classes of locally presentable categories, preprint (1998).

CHK. C. Cassidy, M. Hébert and G.M. Kelly, Reflective subcategories, localizations, and factorization systems, J. Austral. Math, Soc. (Ser. A) 38 (1985) 387-429.

CT. M.M. Clementino and W. Tholen, Tychonoff's Theorem in a category, Proc. Amer. Math. Soc. 124 (1996) 3311-3314.

CGT1. M.M. Clementino, E. Giuli and W. Tholen, Topology in a category: compactness, Portugaliae Math. 53 (1996) 397-433.

CGT2. M.M. Clementino, E. Giuli and W. Tholen, What is a quotient map with respect to a closure operator? Appl. Categorical Structures (to appear).

Di. $\quad$ Y. Diers, Categories of algebraic sets, Appl. Categorical Structures 4 (1996) 329-341.

DG1. D. Dikranjan and E. Giuli, Closure operators I, Topology Appl. 27(1987) 129-143.

DG2. D. Dikranjan and E. Giuli, Compactness, minimality and closedness with respect to a closure operator, in "Categorical Toplogy and its Relations to Analysis, Algebra and Combinatorics", World Scientific (Singapore, 1989) 284-296.

DT. D. Dikranjan and W. Tholen, Categorical Structure of Closure Operators, Kluwer Academic Publishers (Dordrecht, 1995).

Dy. $\quad$ R. Dyckhoff, Categorical cuts, Topology Appl. 6 (1976) 291-295.

GT. E. Giuli and W. Tholen, Openness with respect to a closure operator, Appl. Categorical Structure (to appear).

HI. H. Henriksen and J. R. Ishell, Some properties of compactifications, Duke Math. J. 25 (1958) 83-106.

He1. H. Herrlich, A generalization of perfect maps. Proc. Third Prague Top. Symp. 1971 (1972) 187-191.

He2. H. Herrlich, Perfect subcategories and factorizations, in: "Coll. Math. Soc. Janos Bolyai" (Keszthely, 1972) 387-401.

HeSS. H. Herrlich, G. Salicrup and G. E. Strecker, Factorizations, denseness, separation and relatively compact objects, Topology Appl. 27(1987) 157-169.

Ho. D. Holgate, The Pullback closure and generalizations of perfectness, Appl. Categorical Structures 4 (1996) 107-120.

Js. I.M. James, Fibrewise Topology, Cambridge University Press, (Cambridge, 1989). 
J. G. Janelidze (G. Z. Dzhanelidze), The fundamental theorem of Galois Theory, Math. USSRSb. 64 (1989) 359-374.

JT. G. Janelidze and W. Tholen, Functorial factorization, well-pointedness and separability, J. Pure Appl. Algebra (to appear).

KP. H. P. Künzi and B. A. Pasynkov, Tychonoff compactifications and R-completions of mappings and rings of continuous function, Appl. Categorical Structures (to appear).

P. B. A. Pasynkov, On extension to mappings of certain notions and assertions concerning spaces, in: Mapping and functors (Eds. V. V. Fedorčuk et al.), Izdat. MGU (Moscow, 1984) 72-102 (in Russian).

Pe1. J. Penon, Objects séparés ou compats dans une catégorie, C. R. Acad. Sc. Paris (Série A) 274 (1972) 384-387.

Pe2. J. Penon, Constructions relatives aux objects compats d'une catégorie, C. R. Acad. Sc. Paris (Série A) 275 (1972) 757-760.

T1. W. Tholen, Diagonal theorems in topology and elsewhere, in: Proc. Sixth Prague Topological Symposium 1986, Helderman Verlag (Berlin, 1988) 559-566.

T2. W. Tholen, Maps in general topology and elsewhere, in: Seminarberichte (Hagen, 1998).

Z. A. Zouboff, Herrlich-type embeddings, partial products and compact maps, preprint (1998).

The author gratefully acknowledges partial financial assistance by the University of L'Aquila (within the framework of an exchange agreement York-L'Aquila), by the Natural Sciences and Engineering Research Council of Canada, and from a NATO Collaborative Research Grant while research on this paper was conducted.

This article may be accessed via WWW at http://www.rmi.acnet.ge/hha/ or by anonymous ftp at ftp://ftp.rmi.acnet.ge/pub/hha/volumes/1999/n6/n6.(dvi,ps,dvi.gz,ps.gz)

Walter Tholen tholen@pascal.math.yorku.ca and tholen@ns.univaq.it

Department of Mathematics and Statistics,

York University,

Toronto, Canada M3J 1P3

and

Dipartimento di Mathematica Pura ed Applicata,

Università, 67100 L'Aquila, Italy 